\title{
DIFFUSE [CII] EMISSION IN INTERSTELLAR SPACE
}

\author{
H. Okuda, H. Shibai, T. Nakagawa, H. Matsuhara \\ Institute of Space and Astronautical Science \\ Japan
}

\author{
T. Maihara, K. Mizutani \\ Kyoto University \\ Japan
}

N. Hiromoto

Communication Research Laboratory

Japan

\author{
Y. Kobayashi \\ Institute of Astronomy \\ University of Tokyo \\ Japan
}

\author{
F. J. Low, T. Nishimura \\ Steward Observatory \\ University of Arizona \\ USA
}

\begin{abstract}
An extensive survey of [CII] line emission has been made with a balloon-borne infrared telescope. It has been found that the emission is diffuse and ubiquitously distributed in general interstellar space.
\end{abstract}

\section{INTRODUCTION}

The fine-structure line of [CII] ${ }^{2} P_{3 / 2}-{ }^{2} P_{1 / 2}(157.7 \mu \mathrm{m})$ has been observed in a number of galactic and extragalactic sources. Most observations have been concerned with interface regions between HII regions and molecular clouds. These observations have delineated a new state of interstellar space, the so-called "photodissociation region," which has been theoretically studied by Tielens and Hollenbach (1985). In general, the emission is diffusely distributed and penetrates deep into the molecular clouds; this effect is explained as due to the clumpy nature of the emission regions. Stacey et al. (1985) have also detected the emission widely distributed in the galactic plane. The current status of the observations and theoretical interpretations have been reviewed by Genzel, Harris, and Stutzki (1989).

We have attempted to make a more extensive survey of diffuse [CII] emissions in the galactic plane with a newly developed technique and better observational conditions achieved at balloon altitudes.

\section{OBSERVATIONS AND RESULTS}

The observations were made with a liquid-He-cooled Fabry-Perot spectrometer that has a resolution of about $2200(\lambda / \Delta)$ or $140 \mathrm{~km} / \mathrm{s}$ in velocity. The spectrometer was used on board a balloon telescope of $50 \mathrm{~cm}$ in diameter with a beam size of 3.6'. A frequency-switching method was successfully applied in the observations and was found to be very efficient in detecting extended components of the emissions.

In the two balloon flights made from the National Stratospheric Balloon Facility in Palestine, Texas, on May 24 and June 4, 1988, we have made (1) detailed mappings of the [CII] 
emissions in M17 and NGC 6334, (2) spectroscopic measurements of [CII] line profiles on the ridge of the galactic center region, and (3) a general survey of [CII] emissions in the galactic plane.

Typical results of the observations are summarized as follows:

1. Two-dimensional maps of [CII] intensity distribution have been obtained for the first time for M17 (Matsuhara et al. 1989) and NGC 6334 . In both cases, the emission is extensively distributed and penetrates deeply into the molecular clouds well beyond the boundaries of HII regions. It is interesting that a fairly strong [CII] emission has been found even in one of the far-infrared sources in NGC 6334, which is not associated with any HII region (Nakagawa et al. 1989).

2. Spectral measurements of the [CII] line have been made in the galactic central region between $l=+l-0.7^{\circ}$. Strong emissions have been detected in every region observed, and the observed spectra show wide velocity dispersion as well as Doppler shift due to the galactic rotation. In addition to the galactic center peak, strong intensity peaks have been seen at symmetrical positions $\left(l=+l-0.6^{\circ}\right)$ from the galactic center (Okuda et al. 1989).

3. A general survey of the emissions was attempted by scanning across the galactic plane in sparse grids wider than the beam size. The contour map made by interpolating the observed points is shown in Figure 1, compared to the far-infrared map obtained by $I R A S$. The emissions are widely distributed in the galactic plane and extended to $\pm 1^{\circ}$ of the galactic latitude (Shibai et al. 1989, in preparation).
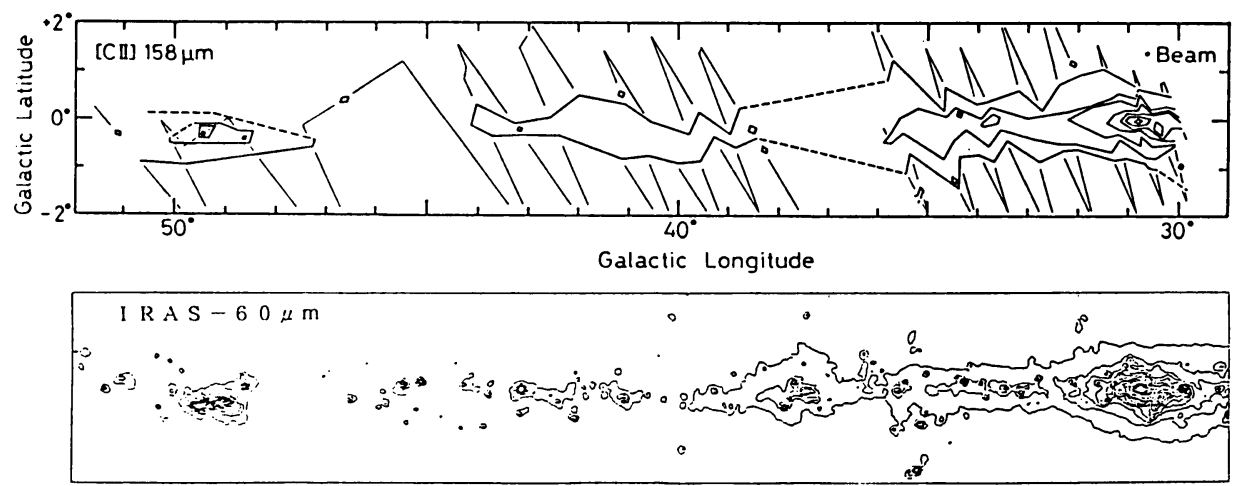

Figure 1. [CII] intensity map in the galactic plane between $l=30^{\circ}$ and $51^{\circ}$ (Shibai et al. 1989, in preparation) in comparison with far-infrared map (IRAS). Thin zig-zag lines represent scanning paths of the observations.

\section{DISCUSSION}

Strong [CII] line emission is generally observed in the HII region and molecular cloud complexes (Stutzki et al. 1988, Matsuhara et al. 1989, Nakagawa et al. 1989, in preparation). The emission is particularly strong at the interfaces between HII regions and molecular clouds, which form "photodissociation regions." The emission pervades deeply into the molecular clouds. This effect is explained by either the clumpy structure of the molecular clouds or the unidentified UV sources distributed uniformly in the clouds. 
However, the most impressive result is that [CII] emissions are extensively distributed even outside the HII region and molecular cloud complexes and ubiquitously in the galactic plane. The contribution of the extended component is energetically dominant over that of the discrete sources, because the latter are stronger in intensity but confined in a small volume. This component may be relevant to the interstellar emissions recently proposed by van Dishoek and Black (1988).

The emitting power of the [CII] line is very large and amounts to $0.3 \%$ of the total farinfrared luminosity. It is embodied in only a single spectral line!

As the HI line $(21 \mathrm{~cm})$ and the $\mathrm{CO}$ line $(2.6 \mathrm{~mm})$ have done, the [CII] line $(158 \mu \mathrm{m})$ would provide an invaluable probe for studies of interstellar space as well as for exploration of the galactic structure.

\section{REFERENCES}

Genzel, R., Harris, A. I., and Stutzki, J. 1989, ESLAB Symposium on Infrared Spectroscopy in Astronomy, Salamanca, ed. Lada, C. L.

Matsuhara, H., Nakagawa, T., Shibai, H., Okuda, H., Mizutani, K., Maihara, T., Kobayashi, Y., Hiromoto, N., Nishimura, T., and Low, F. J. 1989, Ap. J. Letters, 339, L67.

Nakagawa, T., et al. 1989, in preparation.

Okuda, H., et al. 1988, IAU Symposium No. 136: The Center of the Galaxy, ed. Morris, M. p. 145. Dordrecht: Kluwer Academic Publisher.

Shibai, H., et al. 1989, Ap. J., in preparation.

Stacey, G. J., Viscuso, P. J., Fuller, C. E., and Kurtz, N. T. 1985, Ap. J., 289, 803.

Stutzki, J., Stacey, G. J., Genzel, R., Harris, A. I., Jaffe, D. T., and Lugten, J. B. 1988, Ap. J., 332, 379.

Tielens, A. G. G. M., and Hollenbach, D. 1985, Ap. J., 291, 722.

van Dishoek, E. F., and Black, J. H. 1988, Ap. J., 334, 771.

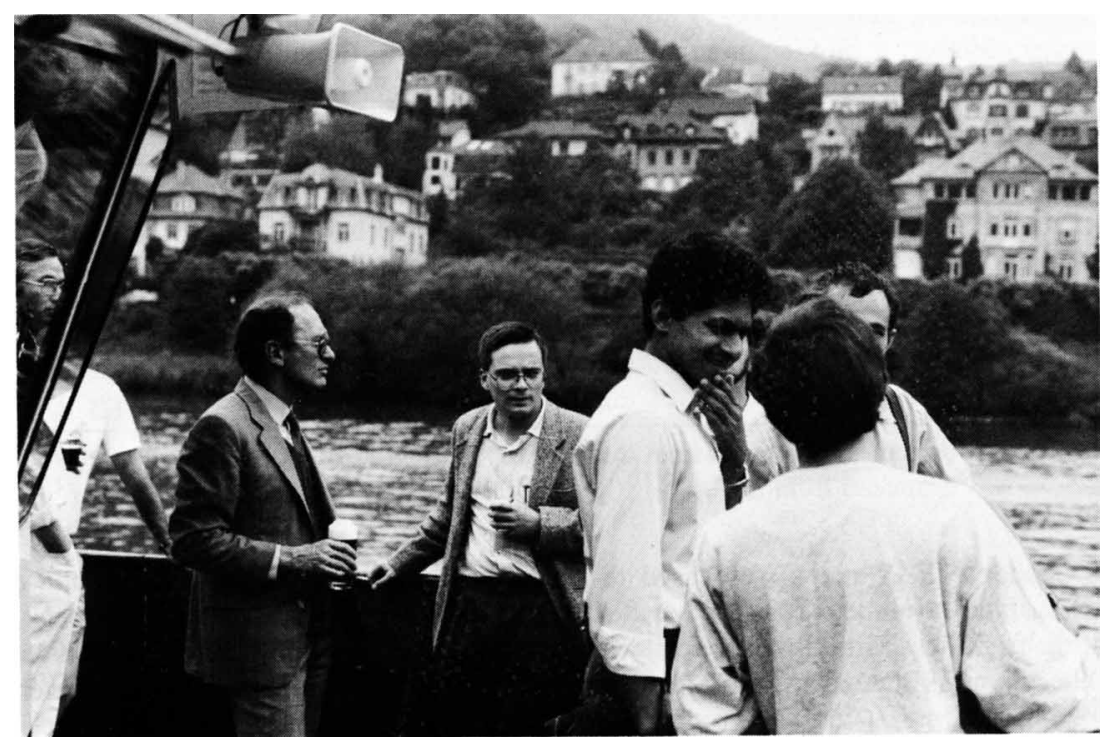

Cruise on the Neckar 